\title{
Fishnet-Based Metamaterials: Spectral Tuning Through Adsorption Mechanism
}

\author{
Z. JAKŠIĆC ${ }^{a, *}$, D. TANASKOVIĆ ${ }^{a}$ And J. MATOVIC ${ }^{b}$ \\ ${ }^{a}$ Institute of Microelectronic Technologies and Single Crystals \\ IHTM - Institute of Chemistry, Technology and Metallurgy, Belgrade, Serbia \\ ${ }^{b}$ Institute for Sensor and Actuator Systems ISAS, Vienna University of Technology, Vienna, Austria \\ In this work we consider the tuning of spectral reflectance of fishnet metamaterials with circular or elliptic \\ apertures due to the presence of dielectric analyte adsorbed within the pores of the dielectric part. We simulated \\ the electromagnetic response of the fishnet metamaterials for the optical range utilizing the finite element \\ method. Our investigation is dedicated to ultrathin, freestanding laminated structures (metal-dielectric-metal \\ self-supported nanomembrane sandwiches with nanometric thickness). We also investigated some issues connected \\ with experimental fabrication of the freestanding fishnets for adsorbtion-based sensors.
}

PACS numbers: 81.05.Xj, 78.67.Pt, 42.25.Bs, 73.20.Mf, 81.07.-b

\section{Introduction}

Since their practical introduction, metamaterials (MM) with negative effective refractive index, also known as left-handed metamaterials, have attracted large attention [1]. Metamaterials generally may be described as artificial metal-dielectric (nano)composites with electromagnetic properties not readily found in nature. Among the various designs proposed for such materials in the optical range, probably the best performance to date has been achieved utilizing the double fishnet structures $[2,3]$. They are negative refractive index MMs with the highest operating frequencies to date, even entering the visible $[4,5]$.

A unit cell of a fishnet metamaterial consists of two square metal sheets in the $x y$ plane separated by a dielectric layer along the $z$ direction. The sheets are discontinued at each edge of the square. Each metal sheet is thus basically a superposition of a thin wire along the $y$ direction and a short slab along the $x$ direction. If a large number of such unit cells is observed simultaneously, the structure has an appearance of a fishnet, where the discontinuities at the edges are the fishnet holes. These holes are typically rectangular, circular or elliptical. A fishnet with circular holes is shown in Fig. 1.

The main advantage of the fishnet structures is their ability to generate left-handed response for a light beam incident perpendicularly to the $x y$ plane, ensuring both lateral and normal coupling. In this manner one obtains a strong left-handed response using a single metal-

\footnotetext{
* corresponding author; e-mail: jaksa@nanosys.ihtm.bg.ac.rs
}

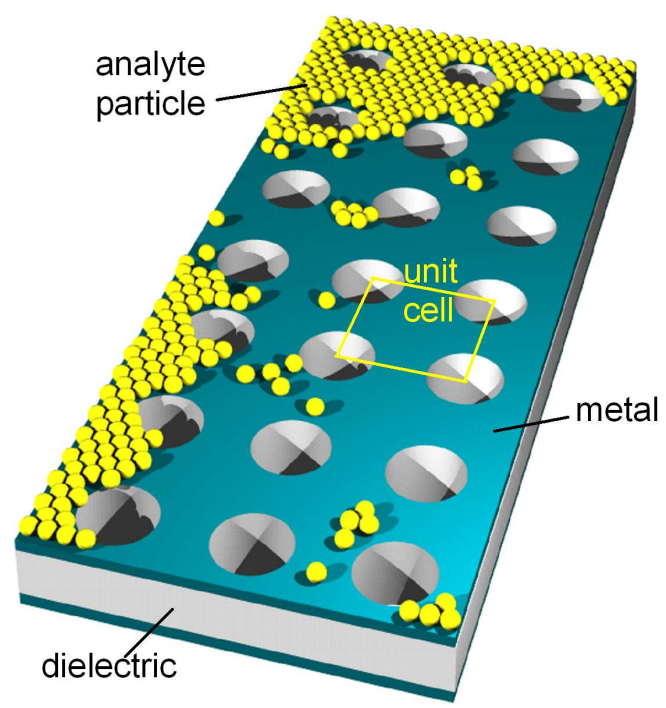

Fig. 1. Fishnet-type double negative metal-dielectricmetal structure. Spheres on the top represent adsorbed analyte particles.

dielectric-metal structure.

It is known that most of the MMs are strongly resonant and their properties are thus very sensitive to even small variations of their geometrical and structural parameters. MM-based sensors have been reported in [6-8]. The tunability of the spectral transmission and reflection of $\mathrm{MM}$ due to adsorption of $\mathrm{nm}$ thin dielectric layer has been described in [6]. This property has a direct practical interest in the use of MM for all-optical sensors of various chemical or biological analytes. In addition to 
that, any resonant metal-dielectric nanocomposite will have its properties strongly modified by adsorption and it is thus of general interest to know the extent of such changes for an MM in a given environment.

In this work we analyzed the behavior of fishnet MM in presence of various dielectric analytes which may be adsorbed within the dielectric part or at the surface of the metal part. We simulated the electromagnetic response of the fishnet MM for the optical range utilizing the finite element method. We considered some issues regarding experimental fabrication of the fishnet MM for adsorbtion-based sensing.

\section{Simulation and results}

Geometry of the unit cell of our fishnet MM is defined in Fig. 2. The width and the length of the unit cell are $a$ and $b$, respectively. The apertures are elliptical, furnishing the minimum widths of the fishnet unit cell $l$ and $w$. The presented geometry shows elliptical holes. In a general case, the hole shape may be circular, elliptical or rectangular.

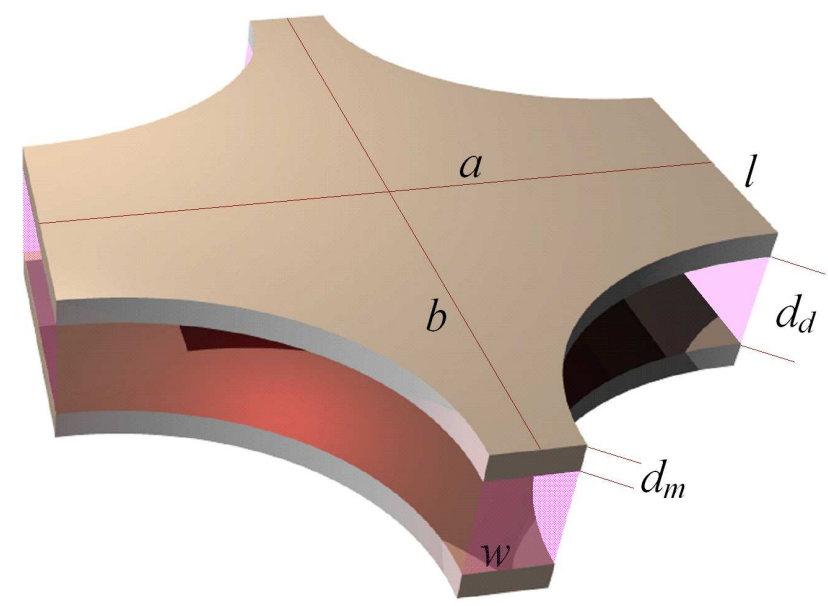

Fig. 2. Geometry of a single unit cell of a fishnet structure.

We calculated the reflection and transmission of a fishnet metamaterial utilizing the finite element method. An illustration of the fishnet MM tunability through physical adsorption within the dielectric part is given in Fig. 3. The dimensions of the structure are given in the inset. The hole shape was circular. The unit cell dimensions were $320 \mathrm{~nm} \times 280 \mathrm{~nm}$. The calculation was done for the case of perfect electric conductor (PEC).

We assumed that the electromagnetic transport characteristics of the fishnet were tuned by changing the relative permittivity of the dielectric layer in the metaldielectric-metal sandwich. No losses were assumed in the dielectric part. Figure 3 shows the shift of the reflectance dip with varying values of the dielectric permittivity.

Changing the angle of incidence of the wave to one perpendicular to the surface (magnetic field in the plane)

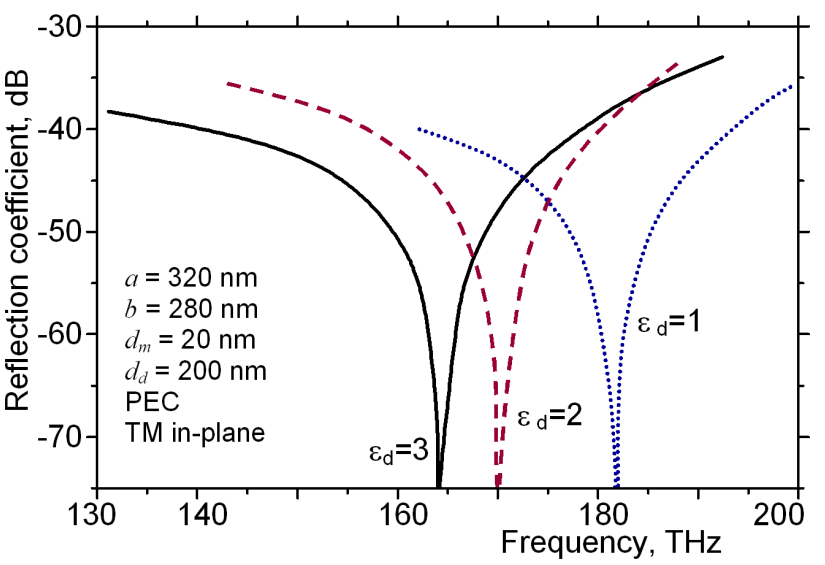

Fig. 3. Reflection from a metal-dielectric-metal fishnet MM with circular holes (geometrical dimensions and adlayer thickness are shown in figure) for the case of PEC.

shifts the peak toward higher frequencies (for instance, the reflectance dip shifts from $170 \mathrm{THz}$ to about $176 \mathrm{THz}$ for a middle layer permittivity of 2 ). Let us note that this calculation, since utilizing PEC, represents an assessment only. Our further simulations showed that the lower conductance of real metals (as in the case of $\mathrm{Ag}, \mathrm{Au}$ ) tends to strongly "smear" the resonant peaks, retaining however the frequency dependence.

\section{Fabrication issues}

In this section we consider the feasibility of the fabrication of adsorption-tunable nanomembrane-based fishnet MMs. As the basic building block of our fishnet MM structures we chose metallic or metal-composite freestanding nanomembranes with large aspect ratio, the structures experimentally fabricated by our team [9] (a SEM photo of a metal-dielectric nanomembrane $8 \mathrm{~nm}$ thick is shown in Fig. 4a). These structures are fabricated by RF sputtering onto a sacrificial silicon substrate and subsequent removal of the substrate through chemical etching to release a freestanding, nanometer thin structure. Our proposal includes the fabrication of laminated structures which consist of good metal ( $\mathrm{Ag}, \mathrm{Au})$, followed by a thicker dielectric layer, to be topped again by metal layer. Thus a structure shown in Figs. 1, 2 can be obtained. The chosen fabrication method allows for deposition of $\mathrm{Au}, \mathrm{Ag}$ and other plasmonic metals, and in the same run the deposition of the dielectric layer.

An alternative approach we considered is to utilize vacuum evaporation for the deposition of the metaldielectric-metal sandwich. This method is less aggressive toward the self-supported ultrathin structure and allows for finer fabrication.

The basic layer in our first experimental implementation consists of non-plasmonic $\mathrm{Cr}$ which acts as a mechanical self-supporting layer for the nanomembrane [9] and in the electromagnetic sense practically behaves as 

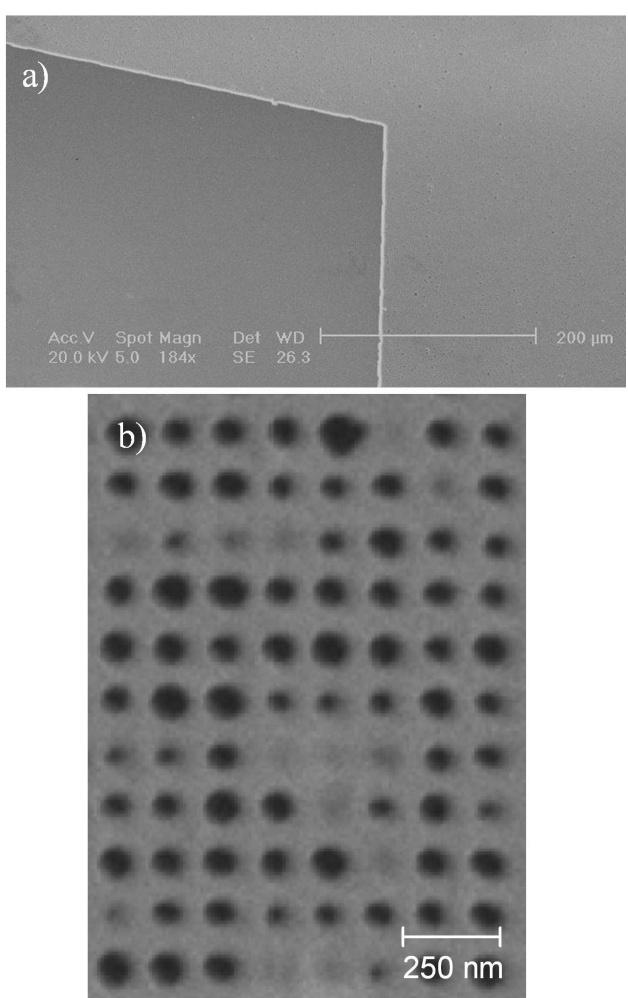

Fig. 4. A building block for the fishnet structure: (a) metal-composite nanomembrane material $8 \mathrm{~nm}$ thick fabricated by sputtering (our concept is to use sandwich containing non-plasmonic $\mathrm{Cr}$ as ultrathin mechanical support and top Au layer as plasmonic material); (b) nanohole array in a metal-composite nanomembrane fabricated by FIB.

a lossy dielectric within the frequency range of interest. The layer is topped by deposited $\mathrm{Ag}$ which ensures plasmonic behavior.

For the fishnet patterning of the structures we chose the focused-ion-beam (FIB) method [10]. A SEM photo of a nanomembrane with an array of nanoholes fabricated by FIB is shown in Fig. 4b. Let us note that the hole size is much smaller than that considered in the previous section. Let us note that this is not a functional fishnet, but a building block only.

\section{Conclusion}

In this paper we considered the feasibility of utilizing fishnet MMs for adsorption-based chemical or biological sensors. We limited ourselves to the structures for the optical range that can be (and some of them already are) fabricated by the state of the art technologies.

The resonant nature of the MM structures, narrow spectral ranges and losses are of no importance for the sensing applications, actually the resonances are even desirable.

In our future work we plan to fabricate a prototype fishnet structure based on laminar self-supported nanomembrane and to characterize it.

\section{Acknowledgments}

This work was funded by the Austrian Science Fund (FWF) within the project L521 "Metalcomposite Nanomembranes for Advanced Infrared Photonics" and by the Serbian Ministry of Science and Technology within the project 11027 "Microsystem and Nanosystem Technologies and Devices".

\section{References}

[1] C.M. Soukoulis, M. Kafesaki, E.N. Economou, Adv. Mater. 18, 1941 (2006).

[2] S. Zhang, W. Fan, N. Panoiu, K. Malloy, R.M. Osgood, S.R.J. Brueck, Phys. Rev. Lett. 95, 137404 (2005).

[3] M. Kafesaki, I. Tsiapa, N. Katsarakis, Th. Koschny, C.M. Soukoulis, E.N. Economou, Phys. Rev. B 75, 235114 (2007).

[4] G. Dolling, M. Wegener, C.M. Soukoulis, S. Linden, Opt. Lett. 32, 53 (2007).

[5] G. Dolling, Ph.D. Thesis, 2007.

[6] Z. Jakšić, O. Jakšić, Z. Djurić, C. Kment, J. Opt. A, Pure Appl. Opt. 9, S377 (2007).

[7] A. Ishimaru, S. Jaruwatanadilok, Y. Kuga, Prog. Electromag. Res. PIER 51, 139 (2005).

[8] C.M. Bingham, H. Tao, X. Liu, R.D. Averitt, X. Zhang, W.J. Padilla, Opt. Express 16, 18565 (2008).

[9] J. Matović, Z. Jakšić, Microel. Eng. 86, 906 (2009).

[10] J. Matović, J. Kettle, E. Brousseau, N Adamovic, in: Proc. 26th IEEE Conf. MIEL, Vol. 1, 2008, p. 104. 